\title{
Métodos univariados y multivariados para analizar el rendimiento académico de la carrera de Ingeniería Agroindustrial en la UNI región norte, Estelí, Nicaragua
}

\author{
Luis María Dicovskiy Riobóo ${ }^{1}$ \\ Manuel Enrique Pedroza Pacheco²
}

\section{RESUMEN}

Con el objetivo de analizar el rendimiento académico de la carrera de ingeniería agroindustrial en la UNI región norte, Estelí, Nicaragua, en el período 2005-2014, fueron implementados diferentes métodos estadísticos univariados y multivariados, tales como: Análisis de Contingencia y Pruebas de Independencia, Prueba " $t$ ” de muestras independientes, Análisis de Correlación de Pearson y Spearman, comparación de promedios, Análisis de Correlación Canoníca y Análisis de Correspondencia Múltiple. En esta investigación se determinaron relaciones estadísticas significativas que han estado influyendo en la calificación del Rendimiento. Los resultados mostraron que el promedio de notas por asignatura, en las Mujeres fue significativamente mejor que el grupo de Varones. Los cursos de matemáticas tuvieron 10 puntos menos que los del perfil social. Hubo correlaciones fuertes y significativas entre las notas de las principales asignaturas agrupadas por afinidad. Las asignaturas de formación básica explicaban las notas de las asignaturas propias de la Profesión. La deserción en aula fue del $16 \%$ en primer año y luego fue decayendo marcadamente. El Análisis de Correspondencia Múltiple de variables cualitativas, mostró que las categorías Mujer y V año estaban más próximo a las categorías de Excelencia y No Desertor, que las categorías Varón, I y II año.

Palabras clave: Rendimiento académico, estadísticas univariadas y multivariadas.

Recibido: 27 de marzo de 2017

Aceptado: 15 abril de 2017

1 Docente UNI Sede Regional Norte, Estelí, estudiante del Doctorado en Gestión y Calidad de la Investigación Científica; UNAN Managua. Correo electrónico: luis.dicovskiy@norte.uni.edu.ni

2 Profesor Titular de UNAN-Managua. Coordinador del Doctorado en Gestión y Calidad de la Investigación Científica. UNAN Managua. Correo electrónico: hppedroza@gmail.com 


\title{
Univariate and multivariate methods to analyze the academic performance of the Agricultiural Engineering program at UNI Norte, Estelí, Nicaragua
}

\begin{abstract}
With the objective of analyzing the academic performance of the agroindustrial engineering career in the northern UNI region, Estelí, Nicaragua, in the period 2005-2014, different univariate and multivariate statistical methods were implemented, such as: Contingency Analysis and Independence Testing, "T" independent sample test, Pearson and Spearman Correlation Analysis, averages comparison, Canonical Correlation Analysis and Multiple Correspondence Analysis. In this investigation, we determined significant statistical relationships that have been influencing the performance score. The results showed that the average grade per subject in the Women was significantly better than the group of Men. The math courses had 10 points less than those of the social profile. There were strong and significant correlations between the notes of the main subjects grouped by affinity. The subjects of basic formation explained the notes of the subjects proper to the Profession. Desertion in the classroom was $16 \%$ in the first year and then declined sharply. The Multiple Correspondence Analysis of qualitative variables, showed that the categories Woman and $\mathrm{V}$ year were closer to the categories of Excellence and Not Deserter, than the categories Male, I and II year.
\end{abstract}

Keywords: Academic performance, univariate and multivariate statistics. 


\section{INTRODUCCIÓN}

Se debe reconocer que sin una educación de calidad no habrá crecimiento económico, equidad ni democracia (Tünnermann Bernhein, 2007). Es sabido que existen diversos y complejos factores objetivos y subjetivos que inciden en la calidad del aprendizaje en los estudiantes universitarios en general y de manera particular en la enseñanza de las ingenierías en Nicaragua, el problema es cómo abordarlos, priorizarlos y luego proponer actividades de mejora.

En los centros escolares se acumulan datos académicos poco aprovechados, y ahí hay un campo útil para los profesionales de la educación donde se podrán hacer cosas interesantes para la propia institución y para otros profesores (Morales Vallejos, 2002, pág. 15).

Las calificaciones, como medida de los resultados de enseñanza, son producto de diferentes condicionantes, tanto de tipo personal del estudiante, como didácticas del docente, contextuales e institucionales, y que todos estos factores median el resultado académico final (Garbanzo Vargas, 2007, pág. 48) . Esto conlleva a afirmar que una de las dimensiones más importantes en el proceso de enseñanza aprendizaje la constituye el "rendimiento académico del alumno" (Ruiz, Ruiz, \& Ruiz, 2010, pág. 1).

La Universidad Nacional de Ingeniería, (UNI), en Nicaragua tiene como finalidad "contribuir a la transformación tecnológica y al desarrollo sustentable de Nicaragua y la región Centroamericana" (Corea, y otros, 2015, pág. 121). La UNI, consciente de su responsabilidad histórica y con apego a su Visión, Misión, Principios y a su profunda vocación social, y dada la demanda creciente de las carreras de ingeniería por los jóvenes bachilleres del norte del país, en marzo de 2005, en Estelí, apertura su primera sede Regional con cuatro carreras. Entre estas estaba Ingeniería Agroindustrial, la primera en su tipo a nivel nacional. La carrera estaba estructurada por 57 asignaturas y un

trabajo de curso, con 214 créditos. El pensum hace énfasis en el desarrollo de capacidades de investigación y en la realización de prácticas profesionales. En el 2005 se inicia a impartir con un grupo de primer año (UNI Sede Regional Norte, 2007).

En el año 2011 se realiza el primer proceso de autoevaluación de la carrera de Ingeniería Agroindustrial con base en el manual de acreditación de la Agencia Centroamericana de Acreditación de Programas de Arquitectura e Ingeniería (ACAAI) y con fines de implementar un plan de mejora que permita acreditar la carrera a futuro ante esta agencia. Según el plan de mejora surgido de la autoevaluación, la carrera cumple con las condiciones para ser acreditada, pero dentro de las actividades pendientes a mejorar está la evaluación y seguimiento del proceso enseñanza aprendizaje para permitir implementar instrumentos que brinden seguimiento a estudiantes de bajo, medio y alto rendimiento (UNI Sede Regional Norte, 2012).

Sin embargo, cuando se calculó Tasa de Egresados en 5 años, estudiantes que han aprobado todas las asignaturas en 5 años, pero les falta su monografía. Partiendo del ingreso 2005 hasta el ingreso 2010. Se observó una tasa de egreso del 40 \%. Cifra que indicó que hubo problemas de deserción o retrasos en egresar a los nuevos profesionales, aspecto que el proceso de autoevaluación no contempló cuando se hizo, ver Cuadro 1.

Cuadro 1. Porcentaje de los Egresados de la carrera de Ingeniería Agroindustrial, UNI Norte1

\begin{tabular}{llr}
\hline Año ingreso & Año Egreso & \% de Egreso en 5 años \\
\hline 2005 & 2009 & 25 \\
2006 & 2010 & 44 \\
2007 & 2011 & 52 \\
2008 & 2012 & 37 \\
2009 & 2013 & 35 \\
2010 & 2014 & 50 \\
\hline Fuente propia & partir de datos del Sistemas de Registro \\
Académico de la Sede UNI Norte
\end{tabular}


De la información anterior se deduce que hace falta investigación que aporte información relevante sobre las causas que han incidido en la Tasa de Egresados de la carrera y en la calidad de los graduandos. Esta es una información que no solo impactará en el futuro estudiante y en su familia, sino también al mismo tiempo incidirá en toda sociedad en general, que pierde la oportunidad de un Ingeniero formado localmente y con capacidad de incidir en el desarrollo tecnológico de la región. Esto además de las altas pérdidas económicas que tiene el país, ya que la formación de ingenieros es de las más caras entre las carreras universitarias.

En esta investigación, basada en las calificaciones por asignatura, se implementan diferentes métodos univariados y multivariados, mediante el diseño de un cuasiexperimento.. Como objetivo general se analizan las relaciones estadísticas que podrían explicar algunas de las posibles causas que han estado influyendo en el número de egresados e indirectamente en la calidad del Rendimiento Académico de la carrera de Ingeniería Agroindustrial de la UNI Región Norte, en el período 2005-2014. De manera particular, se trató de responder mediante análisis estadístico a las siguientes preguntas:

- ¿Han tenido los varones y mujeres un comportamiento diferente en el rendimiento académico?

- ¿Son las asignaturas más complejas en su estudio, como las de matemáticas, evaluadas con menor nota que las asignaturas de menos complejidad como las del tipo social?

- ¿Los estudiantes que llevan mejor promedio en un tipo de a asignaturas, por ejemplo, física matemáticas, también tiene mejor promedio en las asignaturas de otro tipo, por ejemplo, las tecnológicas?

- ¿Están relacionado el comportamiento en las asignaturas de formación básica con las asignaturas de formación profesional?

- ¿La deserción en Aula está influida por el año académico que el estudiante cursa y/o por el género?

\section{MATERIALES Y MÉTODOS}

El Sistema de Registro Académico de la Sede UNI Norte de Estelí facilitó de forma anónima, sin identificar nombres, parte de la base de datos histórica, 2005-2015 de registros académicos de la carrera de Ingeniería Agroindustrial, en formato del software Excel. Donde cada fila era la información académica de la nota final de una asignatura de la carrera. Luego la base de datos original fue trabajada con este software para crear nuevas variables de análisis y ordenarlas de tal manera que puedan luego ser operacionales con un software estadístico. Este proceso permitió diseñar un cuasi experimento. En los diseños cuasi experimentales, como en esta investigación, los sujetos de estudio, los estudiantes, no fueron asignados al azar a los grupos, sino que dichos grupos y sus repeticiones ya estaban prefijados antes del experimento, estos ya estaban formados de forma independiente, (Hernandez, Fernández, \& Baptista, Metodología de la Investigación, 2014, pág. 151).

El software para hacer la mayoría de análisis de los datos estadístico fue InfoStat, 2016, versión profesional, en sus rutinas: Medidas Resumen, Tablas de Frecuencia, Regresión, Correlación, Análisis de Variancia y "Análisis Multivariado" (Di Rienzo J. , y otros, 2015).

Las estadísticas realizadas para responder a las preguntas de investigación fueron: Análisis de contingencia y Pruebas de Independencia, Prueba " $t$ " para inferencia en muestras independientes, Correlaciones de Pearson y Spearman, Comparación de promedios mediante la implementación de un Modelo Lineal Generalizado Mixto y en Estadística Multivariada Correlaciones Canonícas y el gráfico biplot de un Análisis de Correspondencia Múltiple.

Para responder si los varones y mujeres han tenido una conducta diferente académicamente se construyó la variable "asignatura aprobada", calificación o nota 
mayor a 60 puntos en una escala de 1 a 100 puntos. Esto según El Reglamento del Régimen Académico de la Universidad Nacional de Ingeniería, UNI, en su Título 3, Evaluación del Aprendizaje, Arto 25 que define que la escala de calificación de las asignaturas estará comprendida en el rango de cero a cien. La escala de forma cualitativa es la siguiente la siguiente: 0 - 59 Reprobado, 60 - 69 Regular, 70 - 79 bueno, 80 - 89 Muy Bueno, 90 - 100 Excelente (UNI, 2006, pág. 8).

Luego se hicieron Análisis de contingencia, de Frecuencias Absolutas y de Porcentajes de estudiantes aprobados. El Análisis de Contingencia son tablas de frecuencias de variables categóricas, cuando incluye dos variables se dice que tiene dos criterios, en estas la información se organiza en filas y columnas; el cuerpo de la tabla está básicamente constituido por celdas que contienen las frecuencias observadas, en este ejemplo el porcentaje de "aprobación" de asignaturas y "género", varón - mujer, "nij”, los totales marginales de filas y columnas, "ni" y "nj" respectivamente y total general de 17,734 casos. Datos que iban desde el año 2005 hasta el año 2015. Con esta tabla se pudo verificar la independencia de variables por la prueba Chi-cuadrado. La hipótesis de independencia, H0, establecía que la distribución de frecuencias para de aprobación de asignaturas era la misma en ambos géneros; esto equivalía a decir que aprobar, o no, una asignatura es independientemente de ser varón o mujer (Di Rienzo J., y otros, págs. 275-283).

Para conocer cómo había sido el comportamiento de la variable "Nota de los estudiantes aprobados", puntaje final de estudiantes con nota mayor a 60 puntos, y con 14,747 registros disponibles, se hizo una Prueba " $t$ " para inferencia en muestras independientes. Esto sirvió para responder a una hipótesis sobre la esperanza de la variable aleatoria, definida como una diferencia de medias de las muestras, en este caso "Nota de los estudiantes aprobados" y la variable "Género". En este análisis, se quitaron de la base de datos los estudiantes reprobados. Esta prueba asumió que se disponía de dos muestras independientes, cada una de una misma población o distribución. La prueba se considera como una herramienta para la comparación de medias (esperanzas) en dos poblaciones, distribuciones. Una ventaja de utilizar esta prueba ' $t$ ", es que el software InfoStat hace una prueba previa de homogeneidad de varianzas para seleccionar el estadístico " $\mathrm{t}$ " que corresponda, según sean las varianzas heterogéneas u homogéneas (Di Rienzo J. , y otros, 2008, págs. 6970). La hipótesis de esta prueba se expresó como:

- $\mathrm{H} 0: \mathrm{E}\left(\overline{\mathrm{x}}_{\text {Varón }}\right)=\mathrm{E}\left(\overline{\mathrm{x}}_{\text {Mujer }}\right)$, igualdad de medias;

- $\mathrm{H} 1: \mathrm{E}\left(\overline{\mathrm{x}}_{\text {Varón }}\right) \neq \mathrm{E}\left(\overline{\mathrm{x}}_{\text {Mujer }}\right)$, medias diferentes

También se estableció un cuasi experimento bajo la hipótesis de que las Asignaturas de Matemáticas, cuyo contenido corresponde a tres cursos de cálculo, en general se consideran de mayor dificultad que tres asignaturas del tipo social. Con los datos originales se generó una matriz transpuesta, donde cada asignatura generó una variable y cada fila correspondía a las notas de un estudiante dado. De esta nueva matriz, se tomó una muestra de 257 estudiantes, que eran los que habían aprobado seis asignaturas de primero y segundo año de la carrera de Ingeniería Agroindustrial. Luego se crearon dos variables, una con los promedios de las tres asignaturas de matemáticas y la otra con tres asignaturas del tipo social. Las tres asignaturas del ámbito de sociales fueron: Cultura de Paz y Derechos Humanos, Historia de Centroamérica y Redacción Técnica. El grupo de matemáticas estuvo formado por las asignaturas: Matemática I, Matemática II y Matemática III, cuyo contenido es un curso completo de cálculo.

Para responder a la pregunta de, si hay diferencia en la forma de evaluación por estudiante con estos dos grupos, Matemáticas y Sociales, se hizo una prueba ' $\mathrm{t}$ " para inferencia en datos apareadas, no independientes. Esta prueba se usa cuando las esperanzas de dos grupos se obtienen de muestras que están relacionadas; es decir, los resultados del primer grupo no son independientes 
de los del segundo (Di Rienzo J. A., y otros, 2008, pág. 177), en este caso no existe independencia ya que lo que se compara son las notas de un mismo estudiante, en diferentes asignaturas. Para realizar esta prueba "t" de datos apareados, su prueba de hipótesis quedó construida de la siguiente manera:

- $\mathrm{H} 0: \mathrm{E}\left(\mathrm{x}_{\text {Matemáticas }}\right)-\mathrm{E}\left(\mathrm{x}_{-}^{-}\right.$Sociales $)=0$, igualdad de diferencia de medias;

- $\mathrm{H} 1: \mathrm{E}\left(\overline{\mathrm{x}}_{\text {Matemáticas }}\right)-\mathrm{E}\left(\mathrm{x}_{-}^{-}\right.$Sociales $) \neq 0$, no igualdad de medias diferentes, donde E significa Esperanza matemática, promedio.

A fin de establecer relaciones de asociación entre las asignaturas, con los 57 cursos de la carrera de Ingeniería Agroindustrial y con las notas de 209 estudiantes egresados, se generó un matriz transpuesta con 9 agrupaciones de asignaturas, las cuales fueron: Física-Matemáticas, Químicas-Biológicas, Sociales Ambientales, Informáticas, Inglés, Administrativas, de Investigación, de Producción Agrícola, y de Tecnología Agroindustrial. A continuación, en el cuadro 2 se muestran las asignaturas comprendidas dentro de los agrupamientos realizados.

Cuadro 2. Asignaturas y Agrupamientos

\begin{tabular}{ll}
\hline Agrupamientos & Asignatura de la Carrera \\
\hline Administrativas & Administración De Empresa \\
& Agroindustriales \\
& Administración De Operaciones \\
& Administración De Recursos \\
& Humanos \\
& Agro Negocios \\
& Comercio Internacional \\
& Contabilidad Básica y de Costos \\
& Contabilidad Financiera \\
& Desarrollo Empresarial \\
& Economía \\
& Formulación y Evaluación de \\
& Proyectos \\
& Ingeniería Económica \\
& Mercadeo De Productos \\
& Agroindustriales \\
&
\end{tabular}

\begin{tabular}{|c|c|}
\hline \multirow{7}{*}{$\begin{array}{l}\text { Física- } \\
\text { Matemáticas }\end{array}$} & Estadística \\
\hline & Física I \\
\hline & Matemática I \\
\hline & Matemática II \\
\hline & Matemática III \\
\hline & Mecánica de Fluidos \\
\hline & Termodinámica \\
\hline \multirow[t]{2}{*}{ Informática } & Informática I \\
\hline & Informática II \\
\hline \multirow[t]{2}{*}{ Inglés } & Inglés I \\
\hline & Inglés II \\
\hline \multirow[t]{4}{*}{$\begin{array}{l}\text { De } \\
\text { investigación }\end{array}$} & $\begin{array}{l}\text { Metodología de la Investigación } \\
\text { Científica }\end{array}$ \\
\hline & $\begin{array}{l}\text { Seminario Metodológico de } \\
\text { Investigación I }\end{array}$ \\
\hline & $\begin{array}{l}\text { Seminario Metodológico de } \\
\text { Investigación II }\end{array}$ \\
\hline & $\begin{array}{l}\text { Seminario Metodológico de La } \\
\text { Investigación III }\end{array}$ \\
\hline \multirow{7}{*}{$\begin{array}{l}\text { Químicas- } \\
\text { Biológicas }\end{array}$} & Producción Agrícola Pecuaria \\
\hline & Producción Hortofrutícola \\
\hline & Balance de Materia Y Energía \\
\hline & Bioquímica \\
\hline & $\begin{array}{l}\text { Fundamentos de los Procesos } \\
\text { Biológicos }\end{array}$ \\
\hline & Química De Alimentos \\
\hline & Química General \\
\hline \multirow{8}{*}{$\begin{array}{l}\text { Sociales- } \\
\text { Ambientales }\end{array}$} & Cultura De Paz Y Derechos Humanos \\
\hline & Filosofía \\
\hline & $\begin{array}{l}\text { Historia de Centroamérica y } \\
\text { Nicaragua }\end{array}$ \\
\hline & $\begin{array}{l}\text { Humanismo, Cultura Y Valores } \\
\text { Sociales }\end{array}$ \\
\hline & Redacción Técnica \\
\hline & Sociología y Ética \\
\hline & $\begin{array}{l}\text { Tecnología Apropiada Rural y } \\
\text { Transferencia Tecnológica }\end{array}$ \\
\hline & Tecnología Y Medio Ambiente \\
\hline \multirow{6}{*}{$\begin{array}{l}\text { Tecnología- } \\
\text { Agroindustrial }\end{array}$} & Acuicultura \\
\hline & $\begin{array}{l}\text { Buenas Prácticas Agrícolas y de } \\
\text { Manufactura }\end{array}$ \\
\hline & Diseño de Plantas Agroindustriales \\
\hline & Eléctrica y Electrotecnia Fundamental \\
\hline & Gestión y Aseguramiento De Calidad \\
\hline & Higiene y Seguridad Industrial \\
\hline
\end{tabular}


Ingeniería Post-Cosecha I

Ingeniería Post-Cosecha II

Introducción a la Ingeniería

Agroindustrial I

Introducción a la Ingeniería

Agroindustrial II

Manufactura En Conservas

Esterilizadas

Operaciones Mecánicas

Operaciones Unitarias

Proceso Agroindustriales IV

Procesos Agroindustriales I

Procesos Agroindustriales II

Procesos Agro industriales III

Técnicas de Automatización y control

\section{Fuente propia}

Considerando que el tipo de variable a utilizar, las agrupaciones de asignaturas, no eran necesariamente normales, para medir las asociaciones bivariadas de los nueve grupos, se utilizaron dos coeficientes de correlación, uno paramétrico y el otro no paramétrico. El coeficiente de correlación de Pearson, es el más extensamente usado, es una medida de la magnitud de la asociación lineal entre dos variables que no depende de las unidades de medida de las variables originales. El coeficiente de correlación de Spearman es una medida no paramétrica de asociación basada en rangos, que puede ser usado con variables discretas o continuas no necesariamente normales (Di Rienzo J. , y otros, 2008, pág. 145).

Luego con estos 9 agrupamientos de asignaturas se hicieron 2 conjuntos de estudio que fueron sometidos a un Análisis de Correlación Canónica, ACC. El objetivo de esta prueba multivariada, es cuantificar la validez de la relación, entre dos conjuntos de variables (dependiente e independiente) (Badii, Castillo, Cortez, A, \& Villalpando, 2007). Para propósitos descriptivos el ACC, no necesita establecer supuestos sobre la forma de las distribuciones de las variables. Estas variables pueden ser del tipo ordinal o nominal. Sin embargo, si se desea evaluar la significancia de la asociación entre las variables canonícas, debe introducirse el supuesto de multinormalidad de las variables y de homogeneidad de variancias (Hernández Rodríguez, 1988, pág. 77).

El ACC se hizo con 209 casos de estudiantes egresados, calculando correlaciones entre dos grupos de variables y probar su significancia estadística. Para crear los dos grupos, primero se hicieron nueve agrupamientos por afinidad, que consideraron las 57 asignaturas de la carrera. El primer grupo estuvo formado por 5 agrupamientos de asignaturas: Física Matemáticas, Química Biología, Sociales Ambientales, Informáticas e inglés. Este grupo involucraba las asignaturas de Formación Básica General. El segundo grupo que contenía a las asignaturas propias de la profesión, estuvo formado por 4 agrupamientos de asignaturas: Administrativas, de Investigación, de Producción Agrícola, y de Tecnología Agroindustrial.

En la salida de InfoStat para el análisis ACC, se puede observar, cada una de las correlaciones canónicas factibles de calcular, el coeficiente de correlación canónica (R), la proporción de la varianza total explicada por cada par de variables canónicas (R2) y el estadístico (lambda), para probar la hipótesis de dicha correlación (Di Rienzo J. , y otros, 2015, pág. 197)

Para vincular la deserción en Aula con la influencia del año académico, que el estudiante cursa, de primero a quinto, se creó una variable binaria (0 y 1), donde el 0 $=$ desertor y el 1 = No Desertor, esto se hizo con 17,734 filas de datos. Se consideró que un estudiante fue desertor en Aula en una clase dada, cuando la nota final de la asignatura era igual a cero. No se debe confundir esta variable como deserción de la universidad, dato más complejo y difícil de obtener. Sin embargo, los autores de este trabajo observaron que la deserción tomada como abandono a la universidad, cuando se calculó tenía valores cercanos a la deserción promedio en aula. Esta variable, deserción en aula, se analizó con el módulo de Modelos Lineales Generalizados Mixtos de InfoStat. Las comparaciones de medias se hicieron 
por la prueba LSD de Fisher, con un Alfa=0.05 (Di Rienzo, Macchiavelli, \& Casanoves, 2014, págs. 4-5).

Para integrar varias variables cualitativas como: Género en dos categorías Varón-Mujer, Deserción en aula en dos categorías Desertor y No Desertor, Nota en 5 categorías: Reprobado nota 0-59, Regular nota 60-69, Bueno nota 70-79, Muy Bueno nota de 80-89, Excelente notas 90-100, y Año Académico de I a V. Se hizo un gráfico Biplot con una base de datos de 17,734 filas y 4 columnas, bajo el modelo Multivariado de Análisis de Correspondencia Múltiple, ACM, este tipo de análisis multivariado permite explorar tablas multidimensionales de frecuencias.

En un ACM las observaciones multivariadas se grafican en planos para así poder identificar las asociaciones de mayor peso entre las modalidades de varias variables cualitativas. Los resultados del ACM pueden ser representados en un gráfico biplot, donde los puntos, observaciones, muy cercanos en el gráfico, tienen perfil similar. En el gráfico biplot la distancia entre símbolos representando observaciones no tiene interpretación, pero las direcciones de los símbolos desde el origen si pueden ser interpretadas (Di Rienzo J. , y otros, 2008, págs. 220, 226).

Para corroborar lo observado en el biplot, también se hicieron tablas cruzadas con su prueba de independencia Chi-cuadrado. La hipótesis de independencia, H0, establecía que la distribución de frecuencias para Género era la misma para Nota en Categorías y la Deserción en Aula. De igual forma se suponía en H0 que la distribución de frecuencias para el Año Académico era la misma para Nota en Categorías y la Deserción en Aula.

\section{RESULTADOS Y DISCUSIÓN}

Para responder a la pregunta si los varones y las mujeres tienen igual proporción de aprobación de asignaturas, nota mayor a 60 puntos, se construyeron 2 tablas de contingencia. Una tabla de Frecuencias Absolutas y otra de Porcentajes, las cuales se presentan en los cuadros 3 y 4 . En el cuadro 2, se observa que el número de casos consultados fue semejante en número para los varones y las mujeres. En el cuadro 3, se observa un mejor porcentaje de aprobación en las mujeres, en el cuadro 5, se verifica estadísticamente con el valor "p" de la prueba de hipótesis, que nos sitúa en H1, la proporción de aprobados es dependiente del género del estudiante.

Cuadro 3 Frecuencias absolutas de Aprobación por Género. Número de asignaturas aprobadas

\begin{tabular}{llll}
\hline Género & No & Si & Total \\
\hline Mujer & 874 & 7,150 & 8,024 \\
Varón & 2,113 & 7,597 & 9,710 \\
Total & 2,987 & 14,747 & 17,734 \\
\hline
\end{tabular}

Fuente propia

Cuadro 4 Frecuencias Relativas de Aprobación por Género. \% de asignaturas Aprobadas

\begin{tabular}{llll}
\hline Género & No & Si & Total \\
\hline Mujer & 11 & 89 & 100 \\
Varón & 22 & 78 & 100 \\
Total & 17 & 83 & 100 \\
\hline
\end{tabular}

Fuente propia

Cuadro 5. Prueba de Independencia, Aprobación de Asignaturas y Género

\begin{tabular}{llll}
\hline Estadístico & Valor & gl & “p” \\
\hline $\begin{array}{l}\text { Chi Cuadrado } \\
\text { Pearson, X2 }\end{array}$ & 370.54 & 1 & $<0.0001$ \\
\hline
\end{tabular}

Fuente propia

Con la variable, Nota Final de las asignaturas aprobadas, nota mayor a 60 puntos, se hizo una prueba " $t$ ". El p-valor de la prueba " $t$ " rechaza la $\mathrm{H} 0$, de que ambos grupos son iguales. El promedio de notas del grupo de Mujeres fue significativamente mayor en más de tres puntos, que el promedio de notas del grupo de Varones, (cuadro 6). 
Cuadro 6. Prueba T para muestras Independientes, Variable: Nota de Aprobados- Clasificación por Género - prueba: Bilateral

\begin{tabular}{lll}
\hline Estadístico & $\begin{array}{l}\text { Grupo 1 } \\
\text { Mujer }\end{array}$ & $\begin{array}{l}\text { Grupo 2 } \\
\text { Varón }\end{array}$ \\
\hline Valor "n". frecuencia Absoluta & 7,150 & 7,597 \\
Media variable "Notas" & 77.68 & 74.19 \\
p-valor Igualdad de Variancias & 0.0007 & \\
Valor "t" calculado & 18.98 & \\
p-valor prueba "t" & $<0.0001$ & \\
\hline
\end{tabular}

Fuente propia

Vargas M, (2010, pág. 319) en su estudio sobre los factores que determinan el rendimiento académico en matemáticas en la UNI expresa, que "se estima que no existan diferencias significativas entre hombre y mujeres con respecto al rendimiento académico en base de las habilidades intelectuales, (si ocurre) se espera encontrar aspectos sociales propios del contexto que expliquen la conducta diferenciada entre ambos géneros". El comportamiento diferenciado entre géneros también se ha encontrado en otros estudios por ejemplo en Rosario, Argentina, cuando se estudió los determinantes de la deserción universitaria se observó que el género también jugó un papel importante en el abandono de la universidad, un varón tenía una 1.36 veces mayor posibilidad de abandono que el de una mujer (Giovagnoli, 2002, pág. 24).

El hecho que las estudiantes mujeres de la carrera de Agroindustria tengan una menor deserción en aula permite conjeturar varias hipótesis, una de ellas que quizá hubo una mayor motivación a la carrera en este grupo, sin embargo, esto se debe verificar por otros métodos de investigación, por ejemplo, los cualitativos.

Comparando la complejidad de tres asignaturas de Matemática y tres de Sociales bajo una prueba " $t$ " de muestras apareadas, con la variable Notas Final de 257 estudiantes que habían aprobado estas 6 clases, se rechazó la $\mathrm{H} 0$, la diferencia entre los dos grupos es significativa, el grupo de Asignaturas de Sociales fue evaluada con más de 10 puntos de diferencia que los cursos de Cálculo Matemático, (cuadro 7).

Cuadro 7. Prueba T (muestras apareadas), Grupo de Matemáticas versus Grupo de Sociales

\begin{tabular}{|c|c|c|c|c|}
\hline Grupo 1 & Grupo 2 & $\mathbf{N}$ & $\mathbf{T}$ & "P" Bilateral \\
\hline Matemáticas & Sociales & 257 & -20.37 & $<0.0001$ \\
\hline $\begin{array}{l}\text { Media } \\
\text { (1) }\end{array}$ & $\begin{array}{l}\text { Media } \\
(2)\end{array}$ & $\begin{array}{l}\text { Media } \\
\text { (dife- } \\
\text { rencia) }\end{array}$ & & \\
\hline 73.75 & 84.29 & -10.54 & & \\
\hline
\end{tabular}

De los 9 grupos creados a partir de las notas de los estudiantes aprobados en las 56 asignaturas de la carrera de Agroindustria; las asignaturas de Inglés y Sociales-Ambientales tuvieron la mejor valoración en promedio y las de Física y Matemáticas la menor, (cuadro 8).

Cuadro 8. Medidas resumen de los 9 Grupos generados con las 56 Asignaturas de la carrera

\begin{tabular}{llllll}
\hline Variable & n & Media & D.E. & Mín & Máx \\
\hline Administrativas & 209 & 74,83 & 6,70 & 61.0 & 94,3 \\
Física-Matemáticas & 209 & 72,78 & 7,26 & 60,6 & 98.0 \\
Informáticas & 209 & 78,24 & 9,27 & 60.0 & 100 \\
Inglés & 209 & 79,00 & 8,52 & 60.0 & 100 \\
De-Investigación & 209 & 74,73 & 9,19 & 60.0 & 95.0 \\
Producción-Agrícola & 209 & 77,79 & 9,18 & 60.0 & 100 \\
Químicas-Biológicas & 209 & 76,04 & 7,50 & 60,8 & 96,2 \\
Sociales- & 209 & 81,99 & 6,83 & 63,3 & 97,1 \\
Ambientales & & & & & \\
Tecnología- & 209 & 75,81 & 6,94 & 61,3 & 96,0 \\
Agroindustrial & & & & & \\
\hline Fuentepropia
\end{tabular}

Fuente propia

Las correlaciones bivariadas entre los 9 grupos de asignaturas de 209 estudiantes egresados mostraron que en general todos los grupos están asociados positiva y significativamente por las técnicas de correlación Pearson y Spearman. Esto significa que un estudiante que es bueno en sus notas en un grupo de asignaturas, también tiende a tener mejor nota en 
los otros grupos. Hubo tres tipos de correlaciones que se destacan por lo fuerte, estas fueron entre las asignaturas: Administrativas $\mathrm{y}$ las Sociales
Ambientales, de Tecnología Agroindustrial y las Sociales Ambientales, las Física Matemáticas con las de Tecnología Agroindustrial, (cuadro 9).

Cuadro 9. Correlaciones bivariadas de Pearson y Spearman de 9 grupos de Asignaturas

\begin{tabular}{|c|c|c|c|c|c|}
\hline Variable (1) & Variable (2) & $\mathbf{n}$ & Pearson & Spearman & p-valor \\
\hline Administrativas & Física Matemáticas & 209 & 0,75 & 0,72 & $<0,0001$ \\
\hline Administrativas & Informáticas & 209 & 0,50 & 0,47 & $<0,0001$ \\
\hline Administrativas & Inglés & 209 & 0,52 & 0,5 & $<0,0001$ \\
\hline Administrativas & De Investigación & 209 & 0,69 & 0,65 & $<0,0001$ \\
\hline Administrativas & Producción Agrícola & 209 & 0,49 & 0,46 & $<0,0001$ \\
\hline Administrativas & Químicas Biológicas & 209 & 0,65 & 0,58 & $<0,0001$ \\
\hline Administrativas & Sociales Ambientales & 209 & 0,73 & 0,72 & $<0,0001$ \\
\hline Administrativas & Tecnología Agroindustrial & 209 & 0,86 & 0,81 & $<0,0001$ \\
\hline Física Matemáticas & Informáticas & 209 & 0,57 & 0,55 & $<0,0001$ \\
\hline Física Matemáticas & Inglés & 209 & 0,60 & 0,58 & $<0,0001$ \\
\hline Física Matemáticas & De Investigación & 209 & 0,62 & 0,59 & $<0,0001$ \\
\hline Física Matemáticas & Producción Agrícola & 209 & 0,48 & 0,48 & $<0,0001$ \\
\hline Física Matemáticas & Químicas Biológicas & 209 & 0,70 & 0,65 & $<0,0001$ \\
\hline Física Matemáticas & Sociales Ambientales & 209 & 0,67 & 0,71 & $<0,0001$ \\
\hline Física Matemáticas & Tecnología Agroindustrial & 209 & 0,79 & 0,78 & $<0,0001$ \\
\hline Informáticas & Inglés & 209 & 0,68 & 0,68 & $<0,0001$ \\
\hline Informáticas & De Investigación & 209 & 0,41 & 0,39 & $<0,0001$ \\
\hline Informáticas & Producción Agrícola & 209 & 0,41 & 0,4 & $<0,0001$ \\
\hline Informáticas & Químicas Biológicas & 209 & 0,54 & 0,51 & $<0,0001$ \\
\hline Informáticas & Sociales Ambientales & 209 & 0,62 & 0,6 & $<0,0001$ \\
\hline Informáticas & Tecnología Agroindustrial & 209 & 0,63 & 0,62 & $<0,0001$ \\
\hline Inglés & De Investigación & 209 & 0,43 & 0,41 & $<0,0001$ \\
\hline Inglés & Producción Agrícola & 209 & 0,39 & 0,4 & $<0,0001$ \\
\hline Inglés & Químicas Biológicas & 209 & 0,64 & 0,63 & $<0,0001$ \\
\hline Inglés & Sociales Ambientales & 209 & 0,62 & 0,62 & $<0,0001$ \\
\hline Inglés & Tecnología Agroindustrial & 209 & 0,60 & 0,58 & $<0,0001$ \\
\hline De Investigación & Producción-Agrícola & 209 & 0,43 & 0,42 & $<0,0001$ \\
\hline De Investigación & Químicas Biológicas & 209 & 0,44 & 0,39 & $<0,0001$ \\
\hline De Investigación & Sociales Ambientales & 209 & 0,65 & 0,66 & $<0,0001$ \\
\hline De Investigación & Tecnología Agroindustrial & 209 & 0,75 & 0,72 & $<0,0001$ \\
\hline Producción Agrícola & Químicas Biológicas & 209 & 0,54 & 0,51 & $<0,0001$ \\
\hline
\end{tabular}




\begin{tabular}{llllll} 
Producción Agrícola & Sociales Ambientales & 209 & 0,55 & 0,58 & $<0,0001$ \\
Producción Agrícola & Tecnología Agroindustrial & 209 & 0,63 & 0,63 & $<0,0001$ \\
& & & & & $<0,0001$ \\
Químicas Biológicas & Sociales Ambientales & 209 & 0,68 & 0,67 & $<0,0001$ \\
Químicas Biológicas & Tecnología Agroindustrial & 209 & 0,71 & 0,67 & $<0,0001$ \\
\hline
\end{tabular}

Fuente propia

El Análisis de Correlaciones Canónicas, ACC, calculó 4 correlaciones lineales canónicas, ya que, si bien el grupo de Formación Básica General tenía 5 variables, el otro, con las Asignaturas Propias de Profesión, tenía 4 variables. El mejor modelo, la Primera Correlación Lineal, L (1), tuvo un valor de correlación muy alto, $\mathrm{R}$, y el R2=0.81 indicó que el $81 \%$ de la variabilidad estaba siendo explicada por la correlación. Ambos grupos estaban muy vinculados, las notas de las asignaturas de Formación Básica explicaron bien las notas de las asignaturas de Propias de la Profesión. Ver el cuadro 10 de Correlaciones Canónicas.

Cuadro 10. Correlaciones canónicas de los cuatro modelos generados

\begin{tabular}{lllll}
\hline Estadístico & $\mathbf{L ~ ( 1 )}$ & $\mathbf{L ~ ( 2 )}$ & $\mathbf{L ~ ( 3 )}$ & $\mathbf{L ~ ( 4 )}$ \\
\hline $\mathrm{R}$ & 0.9 & 0.34 & 0.2 & 0.08 \\
$\mathrm{R}^{2}$ & 0.81 & 0.11 & 0.04 & 0.01 \\
Lambda & 367.05 & 34.81 & 10.07 & 1.4 \\
gl & 20 & 12 & 6 & 2 \\
p-valor & 0 & 0.0005 & 0.12 & 0,5 \\
\hline
\end{tabular}

Fuente propia

El ACC también facilitó las combinaciones lineales estandarizadas de cuatro modelos, sin embargo, el modelo L (1) es el que se interpreta. En este, en el primer conjunto de Formación Básica. las asignaturas: Sociales Ambientales y las Físicas Matemáticas son las que tuvieron un mayor peso y en el segundo conjunto con las asignaturas Propias de la Profesión, las de mayor peso fueron las de Tecnología Agroindustrial, (cuadro 11).
Cuadro 11. Coeficientes de las combinaciones lineales estandarizadas

\begin{tabular}{lllll}
\hline $\begin{array}{l}\text { Asignaturas Grupo } \\
\text { Formación Básica }\end{array}$ & L (1) & L (2) & L (3) & L (4) \\
\hline Físicas Matemáticas & 0,41 & $-0,92$ & $-0,72$ & 0,89 \\
Químicas Biológicas & 0,14 & 1,37 & $-0,74$ & $-0,31$ \\
Sociales Ambientales & 0,54 & $-0,56$ & 0,56 & $-1,2$ \\
Informáticas & 0,08 & 0,65 & 0,84 & 0,78 \\
Inglés & $-0,06$ & $-0,32$ & 0,21 & 0,06 \\
\hline
\end{tabular}

Grupo de asignaturas Propias de la Profesión

\begin{tabular}{lllll}
\hline Administrativas & 0,16 & $-0,27$ & $-1,98$ & $-0,14$ \\
De Investigación & 0.0008 & $-1,27$ & 0,29 & 0,8 \\
Producción Agrícola & 0,04 & 0,52 & $-0,31$ & 1,15 \\
Tecnología & 0,83 & 0,87 & 1,77 & $-1,22$ \\
Agroindustrial & & & & \\
\hline
\end{tabular}

Fuente propia

El porcentaje de la deserción en aula, nota final igual a cero puntos y el Año Académico, se analizó bajo la rutina de un Modelo Lineal Generalizado. Esto permitió hacer separación de promedios, los resultados se presentan en la figura 1 y se detallan en el cuadro 12. En este gráfico se visualiza que hay diferencias significativas en todos los años académicos y que en los primeros años ocurre la mayor deserción en aula. Esta deserción en aula casi llega al $16 \%$ en primer año y luego va decayendo marcadamente, llegando en el último año de la carrera a valores menores del $4 \%$.

Estos resultados son coincidentes con los observados en un estudio del Ministerio de Educación Nacional de Colombia, junto con el Centro de Estudios sobre 
Desarrollo Económico de la Universidad de los Andes, sobre los factores determinantes de la deserción estudiantil de pregrado en Colombia dio como resultado que "el abandono se produce fundamentalmente en los 4 primeros semestres de la carrera, dato que coincide con estudios realizados en los Estados Unidos y otros países del continente Latinoamericano y el Caribe" (Pineda Báez, 2010, pág. 20).

Un estudio en la Universidad Nacional de Córdoba realizado por Goldenhersch y citado por Merlino y Ayllon, (2016) encontró que, el "primer año de la universidad está fuertemente correlacionado con la mayoría de las variables que muestran la historia preuniversitaria del estudiante, a saber: escuela secundaria, ocupación de los padres, educación de los mismos, sexo del estudiante, con quién vive y cómo costea sus estudios” (Merlino \& Ayllon, 2016, pág. 21).

Cuadro 12. Comparación de promedios con la proporción de deserciones por año académico

\begin{tabular}{llll}
\hline $\begin{array}{l}\text { Año } \\
\text { Académico }\end{array}$ & $\begin{array}{l}\text { Error. } \\
\text { Estándar }\end{array}$ & $\begin{array}{l}\text { Media } \\
\text { Deserciones }\end{array}$ & $\begin{array}{l}\text { Prueba LSD } \\
\text { Prueba LSD } \\
\text { Fisher }\end{array}$ \\
\hline I & 0,03 & 0,16 & A \\
II & 0,05 & 0,12 & \multicolumn{2}{c}{ B } \\
III & 0,06 & 0,09 & \multicolumn{2}{c}{ C } \\
IV & 0,08 & 0,07 & \multicolumn{2}{c}{ D } \\
V & 0,14 & 0,04 & E \\
\hline
\end{tabular}

Medias con una letra común no son significativamente diferentes $(p>0,05)$. F valor: 64,93; Pvalor: $<0,0001$.

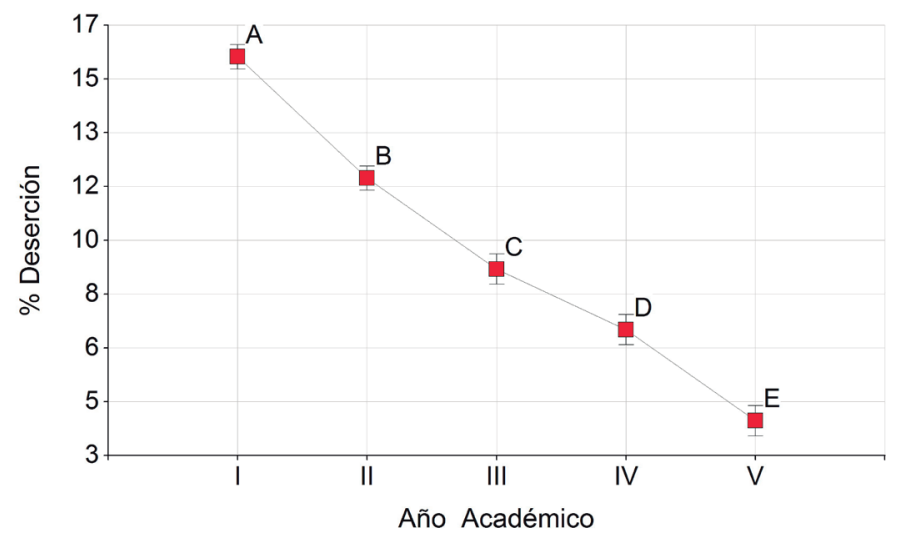

Medias con una letra común no son significativamente diferentes $(p>0.05)$, prueba LSD de Fisher (Alfa=0.05). Las barritas señalan el error estándar de cada promedio.

Figural. Porcentaje de Deserción en Aula y el Año Académico. Fuente propia

Lo anterior se refuerza con lo expresado por Vincent Tinto (1989) quien explica que "la dinámica de la deserción también varía durante el transcurso de la carrera. Las particularidades de las deserciones tempranas son por lo general completamente diferentes de las que se producen en los últimos años. La deserción es no sólo más frecuente en los primeros años de la carrera, sino también más probablemente voluntaria".

Con la rutina de Análisis de Correspondencia Múltiple (ACM), se construyó un gráfico biplot, que integró y relacionó descriptivamente 4 variables cualitativas: Género, Notas en 5 Categorías, Año Académico de I a $\mathrm{V}$ y Desertor en Aula, las cuales se han descrito antes. En la figura 2, se observa por cercanía que la categoría Mujer está más próximo de: Muy Bueno, Excelencia y No Desertor, que la categoría Varón. También V año está más cerca de las categorías Muy Bueno y Excelencia, que I y II año. Las categorías Aplazado y Desertor quedaron muy cerca y separadas del resto. Con este procedimiento, se logró que se vincularan las 4 variables de este estudio en un mismo gráfico.

Las pruebas de Independencia Chi Cuadrado cuyos valores " $p$ " se observan en el cuadro 12, verificaron la falta de independencia de forma altamente significativa, valor "p" < 0.01, de las variables: Género y Año Académico con las variables: Notas en Categorías y Deserción en Aula. 


\section{Biplot de Análisis de Correspondencia}

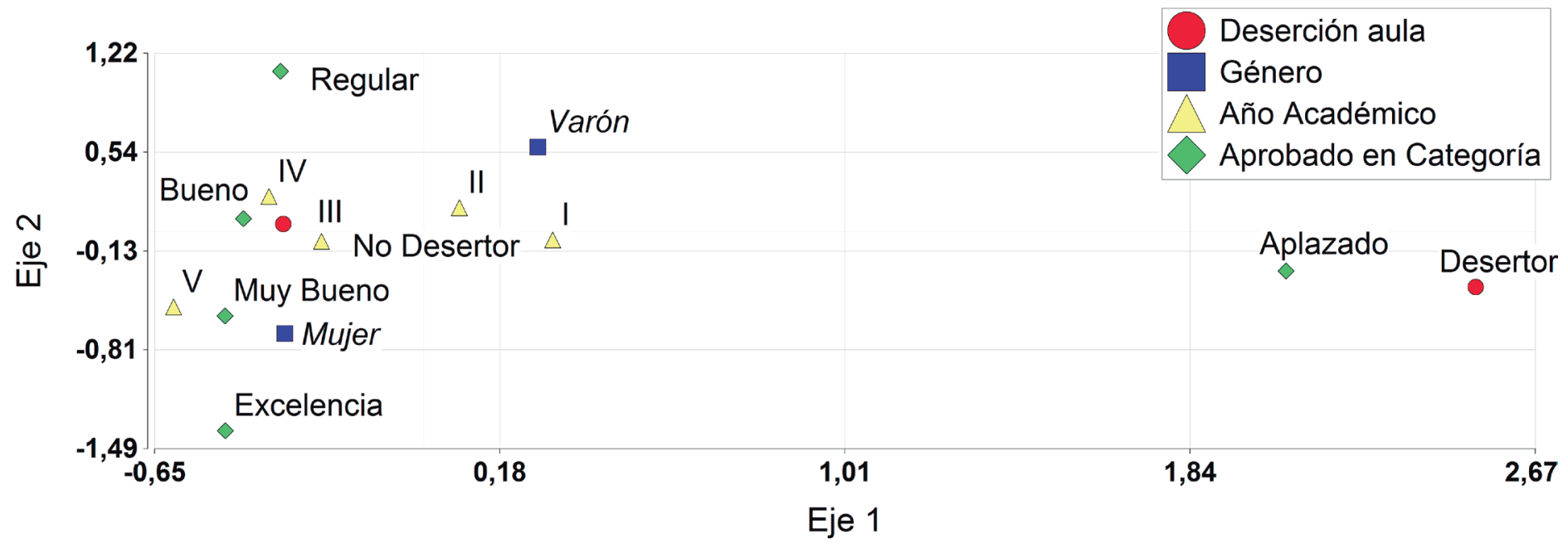

Figura 2. Gráfico Biplot con 4 variables cualitativas. Fuente propia

Cuadro 12. Resultados de las Pruebas de Independencia Chi Cuadrado

\begin{tabular}{lll}
\hline Variable & Variable & $\begin{array}{l}\text { Valor "P" } \\
\text { Prueba X2 }\end{array}$ \\
\hline Género & Notas en Categorías & $<0,0001$ \\
Género & Deserció n en Aula & $<0,0001$ \\
Año Académico & Aprobados en Categorías & $<0,0001$ \\
Año Académico & Deserción en Aula & $<0,0001$ \\
\hline
\end{tabular}

Fuente propia

\section{CONCLUSIONES Y RECOMENDACIONES}

Del análisis de 17,734 notas finales de 11 años de datos, de los cuales 8,024 casos eran de estudiantes mujeres y 9,710 de estudiantes varones, se demostró que las mujeres habían aprobado sus asignaturas en el $89 \%$ de los casos y los varones en el 78 \%. Estas diferencias significativas fueron demostradas mediante la prueba de independencia de X2. Las mujeres tuvieron mayor porcentaje de aprobación. Referente a la variable "nota final por asignatura del estudiante aprobado" comparándose 7,150 casos de mujeres contra 7,597 casos de varones, las mujeres tuvieron 3.49 puntos más de promedio. Estas diferencias significativas fueron demostradas mediante la prueba de " $\mathrm{t}$ " de muestras independientes. Las mujeres obtuvieron mejor porcentaje de aprobación y mayor nota final en las asignaturas aprobadas, que los varones. Esta conducta particular, podría estudiarse con métodos de investigación cualitativa, bajo una hipótesis de que hubo una motivación diferenciada por género hacia la carrera de estudio.

Con una muestra de 257 estudiantes, en una prueba " $t$ " apareada, se comparó el promedio de las notas finales de tres asignaturas de matemáticas contra el promedio de tres asignaturas de tipo social. Se demostró que las notas de las asignaturas de sociales habían tenido una diferencia a su favor de 10.54 puntos, y que esta diferencia fue significativa estadísticamente. A partir de estos resultados se puede inferir que los estudiantes bajo los cursos de cálculo matemático tuvieron un mayor grado de dificultad en su evaluación, que con los cursos del tipo social.

Cuando se agruparon las notas finales de 209 estudiantes egresados, de las 57 asignaturas de la carrera de Ingeniería Agroindustrial en 9 grupos afines, estos se correlacionaron de forma bivariada por los procedimientos de Pearson y Spearman. Los resultados mostraron una positiva y alta correlación significativa entre los grupos de asignaturas: Administrativas con las Sociales Ambientales, Tecnología Agroindustrial con las Sociales Ambientales y las de Física 
Matemáticas con las de Tecnología Agroindustrial. Estas asociaciones dan un hilo conductor entre las asignaturas del tipo Social Ambiental con las propias del ejercicio de la profesión como son las del tipo Administrativo y las Tecnológicas.

Con los mismos nueve agrupamientos de asignaturas, se crearon dos conjuntos de datos, uno con las asignaturas de Formación Básica y el otro con las Asignaturas Propias de la Profesión. Al someter a ambos grupos a un Análisis de Correlaciones Canónicas, se demostró una alta correlación canónica, y que el $81 \%$ de la variabilidad del modelo, estaba siendo explicada por la correlación. Esto confirmó lo observado con las correlaciones bivariadas, aquellos estudiantes que hubieron tenido buenas notas en las asignaturas básicas fueron candidatos a tener buenas notas en las asignaturas tecnológicas.

Con la variable binaria, deserción en aula y 17,734 datos de las 57 asignaturas de la carrera, se estudió la deserción por año académico, de I a V, de la carrera en estudio, con un modelo lineal generalizado, bajo una prueba X2 y separación de medias por la prueba LSD de Fisher. Se demostró que la mayor deserción ocurrió el primer año y la menor en quinto año. Además, hubo diferencias significativas en todos los años, de forma decreciente de primero a quinto. Para entender la alta deserción en aula de primer año se recomienda estudiar la historia preuniversitaria de estos estudiantes.

Se construyó un gráfico biplot mediante un Análisis Multivariado de Correspondencia Múltiple, encontrándose que la categoría Mujer estaba más próximo de Muy Bueno, Excelencia y No Desertor, que la categoría Varón. También se determinó que el $\mathrm{V}$ año está más cerca de las categorías: Muy Bueno, Excelencia y No Desertor, que I y II año. Mostrándose en un sólo gráfico que las mujeres tuvieron un mejor comportamiento en la No Deserción y en la Nota de las asignaturas y que algo semejante ocurrió con los estudiantes de los años superiores.
Los diferentes métodos univariados y multivariados aplicados en este estudio, permitieron demostrar si está ocurriendo o no un fenómeno, pero no son métodos adecuados para responder a la pregunta ¿por qué ocurrió el fenómeno?. El análisis estadístico realizado respondió adecuadamente a las preguntas particulares del estudio, pero no explicó las conductas ocurridas

\section{REFERENCIAS BIBLIOGRÁFICAS}

Badii, M., Castillo, J., Cortez, K., A, W., \& Villalpando, P. (2007). Análisis de correlación canónica (ACC) e investigación científica. (S. N. UANL, Ed.) InnOvaciOnes de NegOciOs, 4(2), 405-422. Recuperado el 06 de Noviembre de 2016, de http:// www.web.facpya.uanl.mx/rev_in/Revistas/4.2/ A9.pdf

Corea, N., Bolaños, E., Pedroza, M. E., Rosales, B., González, E., Dávila, L., . . . Zamora, Z. (2015). La Gestíon de la Investigación en la Educación Superior en Nicaragua. En J. Gairín Sallán, D. Castro Ceacero, \& H. Medrano Rodriguez, La Gestíon de la Investigación en la Educación Superior en Iberoamérica (págs. 119-136). Santiago de Chile, Chile: EDO - UAB - Visión Consultores Ltda.

Di Rienzo, J. A., Casanoves, F., Gonzalez, L. A., Tablada, E. M., Díaz, M. d., Robledo, C. W., \& Balzarini, M. G. (2008). Estadística para las Ciencias Agropecuarias (Séptima ed.). Córdoba, Argentina: Brujas.

Di Rienzo, J., Casanoves, F., Balzarini, M., Gonzalez, L., Tablada, M., \& Robledo, C. (2008). InfoStat, versión 2008. Manual del Usuario. Cordoba, Argentina: Grupo InfoStat, FCA, Universidad Nacional de Córdoba,.

Di Rienzo, J., Casanoves, F., Balzarini, M., Gonzalez, L., Tablada, M., \& Robledo, C. (2015). InfoStat versión 2015. Grupo InfoStat, FCA, Universidad Nacional de Córdoba. Córdoba, Argentina. Obtenido de URL http://www.infostat.com.ar

Di Rienzo, J., Macchiavelli, R., \& Casanoves, F. (2014). Modelos Lineales Generalizados Mixtos 
Aplicaciones en InfoStat. Cordoba: Grupo InfoStat.

Garbanzo Vargas, G. M. (2007). Factores asociados al rendimiento académico en estudiantes universitarios, una reflexión desde la calidad de la Educación Superior Pública. Revista Educación, 3(1), 43-63.

Giovagnoli, P. I. (2002). Determinantes de la deserción y graduación universitaria: Una aplicación utilizando modelos de duración. Universidad nacional de la Plata. Rosario: Departamento de Economía, Facultad de Ciencias Económicas . Recuperado el 14 de Noviembre de 2016, de http:// www.depeco.econo.unlp.edu.ar/doctrab/doc37.pdf Hernández Rodríguez, O. (1988). Temas de Análsis de Estadística Multivariada. San José, Costa Rica: Universidad de Costa Rica.

Hernandez, R., Fernández, C., \& Baptista, P. (2014). Metodología de la Investigación (Sexta ed.). México: MacGraw-Hill.

Merlino, A., \& Ayllon, S. (2016). Experiencias en Investigación Educativa. Córdoba, Argentina: Brujas.

Morales Vallejos, P. (15 de Enero de 2002). La evaluación académica: conceptos y planteamientos básicos. Cuaderno Monográficos del ICE. Serie Didáctica Número 2, 52.

Pineda Báez, C. (2010). La voz del estudiante. El éxito de programas de retención universitaria. Chia,
Colombia: Universidad de La Sabana.

Ruiz, G., Ruiz, J., \& Ruiz, E. (25 de Abril de 2010). Indicador global de rendimiento. (OEI, Ed.) Revista Iberoamericana de Educación (52), 11.

Tinto, V. (1989). Definir la Deserción, una cuestión de perspectiva. Revista de Educación Superior, 71, 33-51. Recuperado el 30 de Agosto de 2016, de Publicaciones ANUIES: http://publicaciones. anuies.mx/pdfs/revista/Revista71_S1A3ES.pdf

Tünnermann Bernhein, C. (2007). La universidad necesaria para el siglo XXI. Managua, Nicaragua: Hispamer/ UPOLI. Recuperado el 19 de Julio de 2016, de http://sajurin.enriquebolanos.org/vega/ docs/2986-1.pdf

UNI. (2006). Reglamento del Régimen Académico de la Universidad Nacional de Ingeniería. Managua: UNI.

UNI Sede Regional Norte. (2007). Plan de Carrera de la Carrera de Agroindustria. Estelí: UNI.

UNI Sede Regional Norte. (2012). Proceso de Autoevaluación del Programa de Ingeniería Agroindustrial. Estelí: UNI.

Vargas Hernández, M. (2010). Actores que determinan el rendimiento académico en matemáticas en la universidad nacional de ingeniería, Nicaragua: un estudio multinivel $y$ de ecuaciones lineales estructurales. Universidad de Costa Rica. San José: Ciudad Universitaria Rodrigo Facio, Costa Rica. 